\title{
Cascaded energy landscape as a key driver for slow yet efficient charge separation with small energy offset in organic solar cells
}

Shin-ichiro Natsuda ${ }^{1}$, Toshiharu Saito ${ }^{1}$, Rei Shirouchi ${ }^{1}$, Yuji Sakamoto ${ }^{1}$, Taiki Takeyama ${ }^{1}$, Yasunari Tamai $^{1,2^{*}}$, Hideo Ohkita ${ }^{1}$

${ }^{1}$ Department of Polymer Chemistry, Graduate School of Engineering, Kyoto University, Katsura, Nishikyo, Kyoto 615-8510, Japan

${ }^{2}$ Japan Science and Technology Agency (JST), PRESTO, 4-1-8 Honcho Kawaguchi, Saitama 3320012, Japan

\section{Corresponding Author:}

*E-mail: tamai@photo.polym.kyoto-u.ac.jp 
Abstract. Recent studies have shown that efficient free carrier (FC) generation with a small voltage loss can be achieved in organic solar cells (OSCs); however, the photophysical insights underpinning this remain unclear. Herein, we examined the mechanisms underlying the FC generation in a stateof-the-art OSC consisting of PM6 and Y6 as an electron donor and acceptor, respectively, wherein the energy offset between the lowest excited singlet state and the charge transfer state is as small as $\sim 0.1 \mathrm{eV}$. We used transient absorption spectroscopy to track the time evolution of electroabsorption caused by electron-hole pairs generated at donor/acceptor interfaces. After hole transfer from Y6 to PM6, we observed slow yet efficient spatial charge dissociation on a time scale of picoseconds. Based on temperature dependence measurements, we found that this slow yet efficient FC generation is driven by downhill energy relaxation of charges through the energy cascade generated near the interfaces. 


\section{Introduction}

Development of novel nonfullerene acceptors (NFAs) has enabled the successful fabrication of efficient polymer/NFA-based organic solar cells (OSCs) $)^{1-5}$. Thus far, polymer/NFA-based OSCs have reached $>18 \%$ power conversion efficiency $(\mathrm{PCE})^{6-9}$, thereby rekindling interest in this research field. Among them, a state-of-the-art OSC consisting of PM6 as a donor polymer and Y6 as a NFA (Figures 1c and 1d) has simultaneously exhibited a relatively high PCE of $>15 \%$ and a small energy loss of $\sim 0.55 \mathrm{eV}^{10}$. In this respect, many studies have been conducted in order to reveal the origin of the success of the PM6/Y6 blend OSCs ${ }^{11-19}$. However, the photophysical mechanisms underlying efficient free carrier (FC) generation in this blend system remain the subject of continuing debate.

OSCs require a donor/acceptor (D/A) interface to dissociate excitons into charges because of the low dielectric constants of organic materials. Upon photoexcitation, singlet excitons are generated in either the D or A material and quickly diffuse to the D/A interface. Thereafter, depending on which material is photoexcited, either an electron or a hole is transferred to its counterpart material, forming a charge transfer (CT) state at the D/A interface. If the electrons and holes that constitute the CT state dissociate beyond their Coulomb capture radius (typically $4-5 \mathrm{~nm}$ considering the entropic contribution to the Gibbs free energy) ${ }^{20}$, they become FCs and can survive up to microseconds, which is long enough for charges to be collected to their respective electrodes. Historically, it was presumed that a large offset between the lowest excited singlet state energy $E_{\mathrm{S} 1}$ and the CT state energy $E_{\mathrm{CT}}$ of 
$>0.3 \mathrm{eV}$ is required for efficient $\mathrm{FC}$ generation in $\mathrm{OSCs}^{20-24}$. For example, Friend and his co-workers have elucidated that this excess energy is required to access higher-energy delocalized states, wherein charges can move quickly away from the D/A interface on a timescale of tens to a few hundreds of femtoseconds ${ }^{25-29}$. On the other hand, in the absence of the offset energy (or the absence of a delocalized state in less-aggregated blends), the CT state is trapped at the interface and finally undergoes geminate recombination to the ground state. The large energy offset prerequisite for efficient $\mathrm{FC}$ generation significantly limits the PCE because lowering $E_{\mathrm{CT}}$ relative to $E_{\mathrm{S} 1}$ reduces the maximum achievable open-circuit voltage $\left(V_{\mathrm{OC}}\right)^{30-33}$. Therefore, efficient FC generation without an energy offset is necessary to reduce the voltage loss and hence, improve the PCE further.

Very few studies have reported polymer/fullerene-based OSCs that exhibit efficient FC generation with small energy offset ${ }^{34-36}$. In contrast, recent studies have shown that efficient FC generation with small energy offset can be achieved in various NFA-based OSCs. Several key ideas have been proposed for the FC generation mechanisms in systems with small energy offset. For example, Menke et al. proposed that a low level of energetic disorder, which corresponds to an Urbach energy of $<30$ $\mathrm{meV}$, is a key to efficient FC generation in small offset systems ${ }^{37}$. The PM6/Y6 blends fulfil this empirical requirement. On the other hand, Karuthedath et al. claimed that the ionization energy (IE) offsets are often underestimated in $\mathrm{OSCs}^{38}$. By performing ultraviolet photoelectron spectroscopy (UPS) measurements, they showed that the IE offset in the PM6/Y6 blend was as large as $700 \mathrm{meV}$ 
and proposed that a large IE offset of $>500 \mathrm{meV}$ is required to attain an internal quantum efficiency of $>80 \%$ because of the interfacial energy level bending caused by electrostatic effects, which in turn are caused by the large quadrupole moments of A-D-A-type NFAs. However, due to the lack of direct experimental evidence, further studies are necessary to fully understand the FC generation mechanisms in the PM6/Y6 blend system.

Herein, we examine the FC generation mechanisms in the PM6/Y6 blend system using transient absorption (TA) spectroscopy. We track the time evolution of electroabsorption (EA) caused by the dipolar electric field generated by an electron-hole pair that constitute a CT state. We show that, after hole transfer from Y6 to PM6, FCs are generated slowly yet efficiently on a time scale of $\sim 10 \mathrm{ps.} \mathrm{This}$ is in sharp contrast to the FC generation dynamics after photoexcitation of PM6, wherein rapid spatial separation is observed, as in the case of previously reported large offset systems. Importantly, despite the slow time scale, FC generation after the hole transfer is as efficient as that after the electron transfer and no thermal activation barriers exist for the FC generation. We find that charge dissociation is driven by downhill energy relaxation of charges through the energy cascade generated near the D/A interface (Figure 1a). 


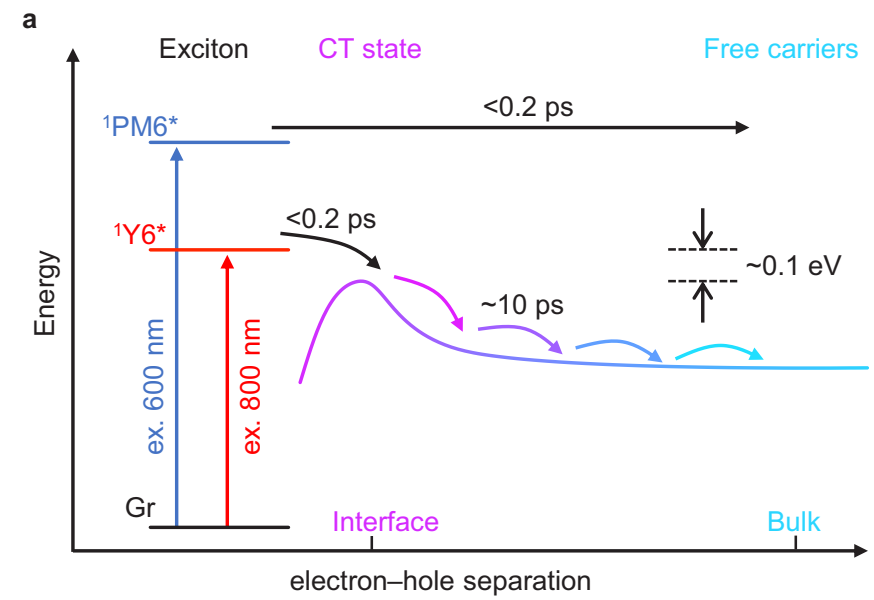

b
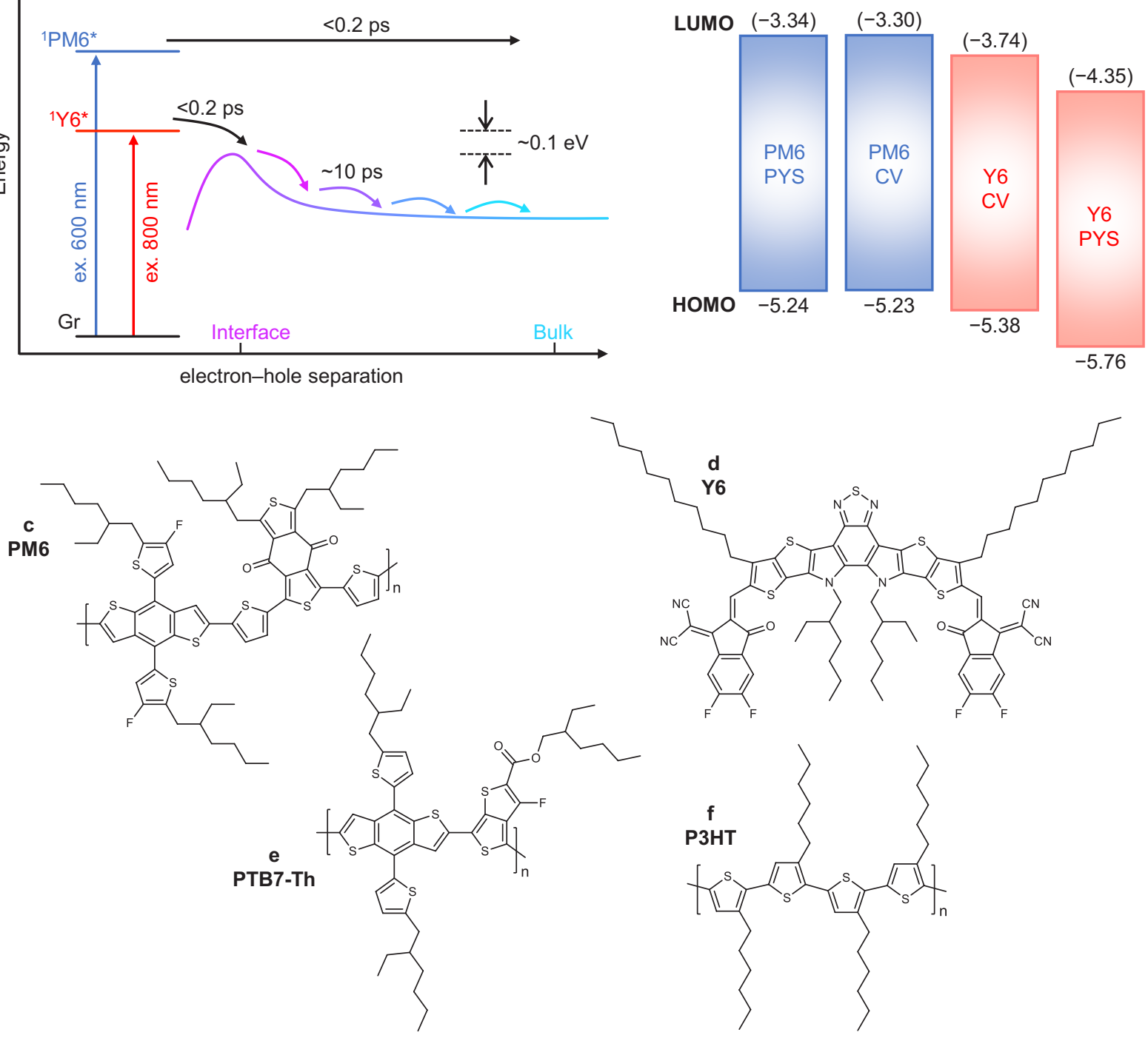

Figure 1. Understanding the charge separation mechanisms with a small energy offset. a

Schematic showing FC generation mechanisms in the PM6/Y6 blend films. Upon photoexcitation at $800 \mathrm{~nm}$, spatial dissociation of electron-hole pairs occurs slowly yet efficiently without an activation barrier, despite the small energy offset of $\sim 0.1 \mathrm{eV}$, driven by downhill energy relaxation of charges through the energy cascade near the interfaces. In contrast, rapid spatial separation on a sub-picosecond time scale occurs after photoexcitation at $600 \mathrm{~nm}$. b HOMO energy levels of PM6 and Y6 determined by CV or PYS. The values in parentheses are the LUMO energy levels calculated as a sum of HOMO and $E_{\mathrm{S} 1}$. Therefore, the LUMO values only serve as a rough estimate for relative comparison. c-f Chemical structures of materials employed in this study. 


\section{Results}

Energy offset in the PM6/Y6 blend systems. Chemical structures of materials employed in this study, steady-state absorption, photoluminescence (PL) and electroluminescence (EL) spectra, the highest occupied molecular orbital (HOMO) energy levels, current density-voltage $(J-V)$ characteristics, and external quantum efficiency (EQE) are found in Figures 1, 2 and in the Supplementary Information.

The absorption and PL spectra of Y6 slightly blue-shift when blended with PM6, indicating that Y6 is slightly less ordered in the blend film than in the pristine Y6 film (Figure 2). In the blend film, $E_{\mathrm{S} 1}$ of Y6 is $1.41 \mathrm{eV}$ (Figure S2), which is slightly higher than that of the pristine Y6 film $(1.39 \mathrm{eV})^{39}$. As shown in Figure 2b, a new PL band clearly appears for the PM6/Y6 blend. This band is not observed for the PTB7-Th/Y6 blend (Figure S6), indicating that it is attributable to CT emission of the PM6/Y6 blend. By applying the Marcus fitting to the CT emission ${ }^{40,41}$, we obtained $E_{\mathrm{CT}}$ of $1.29 \mathrm{eV}$ (details can be found in the Supplementary Information). Therefore, the PM6/Y6 system apparently has a small energy offset of $\sim 0.1 \mathrm{eV}$.

We measured the difference in HOMO energy between PM6 and Y6 using two different methods, as summarized in Figure 1b. The HOMO energy offset derived from cyclic voltammetry $(\mathrm{CV})$ in the solution state was $0.15 \mathrm{eV}$ (Figure $\mathrm{S} 7$ ), consistent with the energy offset between $E_{\mathrm{S} 1}$ and $E_{\mathrm{CT}}$. In contrast, the IE offset derived from photoelectron yield spectroscopy (PYS) in the solid state was as large as $0.52 \mathrm{eV}$ (Figure S8), which is significantly larger than that derived from CV measurements 
in the solution state. These results indicate that the HOMO energy level and hence, the lowest unoccupied molecular orbital (LUMO) energy level, of Y6 depends significantly on its morphology. In other words, Y6 in crystalline states has a deeper HOMO and LUMO energy levels than that in amorphous states. We consider that the large difference in the HOMO and LUMO energy level of Y6 depending on the morphology is a key for efficient FC generation with a small offset, as will be discussed later.
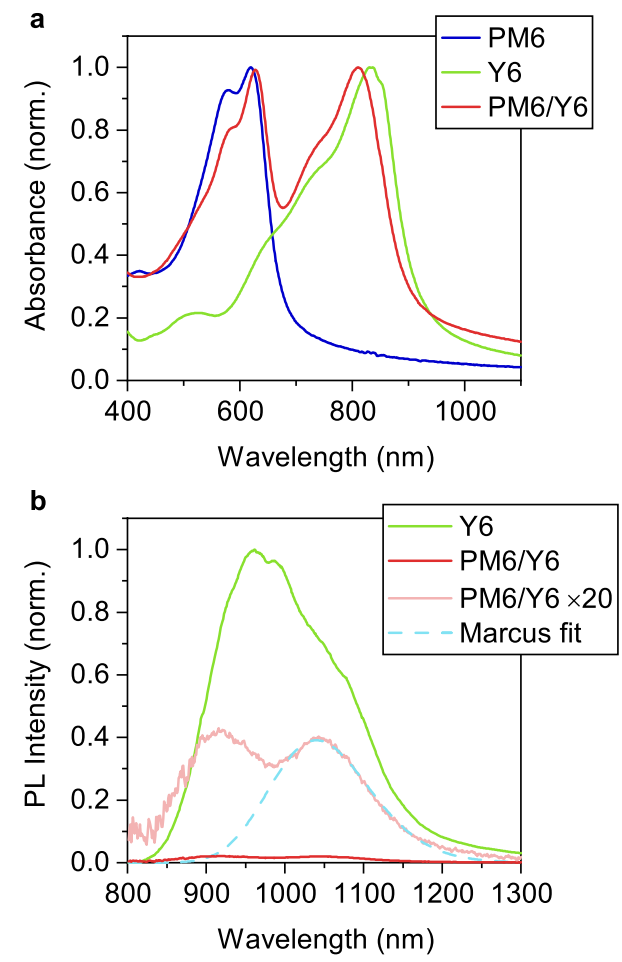

Figure 2. Absorption and PL spectra of an optimized PM6/Y6 blend film. a Steady-state absorption spectrum of an optimized PM6/Y6 blend film as well as pristine PM6 and Y6 films. b PL spectrum of the PM6/Y6 blend film as well as a pristine Y6 film. The broken line represents emission from the CT states obtained by the Marcus fitting. 
TA spectra after Y6 selective excitation. In order to study the charge dissociation dynamics, we performed TA measurements for the optimized PM6/Y6 blend film (Figures 3a and 3b). Here, the excitation wavelength was set to $800 \mathrm{~nm}$ to selectively excite the lower-bandgap Y6. The excitation fluence was kept as low as possible to reduce undesirable bimolecular processes. At an excitation fluence of $1.4 \mu \mathrm{J} \mathrm{cm} \mathrm{cm}^{-2}$, singlet-singlet annihilation (SSA) can be ignored (excitation-fluence dependence is shown in Figure S13). By comparing the TA spectra of the blend with those of a pristine Y6 film, the positive photoinduced absorption (PIA) band observed immediately after photoexcitation at $\sim 930 \mathrm{~nm}$ and the broad PIA tail above $1200 \mathrm{~nm}$ are assigned to singlet excitons of Y $6^{18,39}$. Negative signals in the $750-850 \mathrm{~nm}$ region and sub-650 $\mathrm{nm}$ regions are attributed to the ground-state bleaching (GSB) of Y6 and PM6, respectively, because the positions of these signals coincide with their steady-state absorption spectra. The initial peak position of the Y6 GSB is slightly blue-shifted compared to that of the pristine Y6 film (Figure 3c), which is consistent with the aforementioned slightly blue-shifted steady-state absorption. Singlet excitons of Y6 decayed on a time scale of picoseconds, whereas a new PIA peaking at $\sim 970 \mathrm{~nm}$ remained over nanoseconds after photoexcitation. This broad PIA was still observed on the microsecond time scale (Figure S12) and hence, can be assigned to a superposition of PM6 hole polarons and Y6 anions (details of assignments are described in the Supplementary Information, Figures S11, S12, and S19). This means that charges are generated through hole transfer from Y6 to PM6. The blue line in Figure 3d represents the exciton 
dissociation kinetics monitored at $1050 \mathrm{~nm}$. The rise kinetics was fitted using the sum of two exponential functions with a constant fraction, giving an average rise time constant of $\sim 6.0 \mathrm{ps.} \mathrm{Note}$
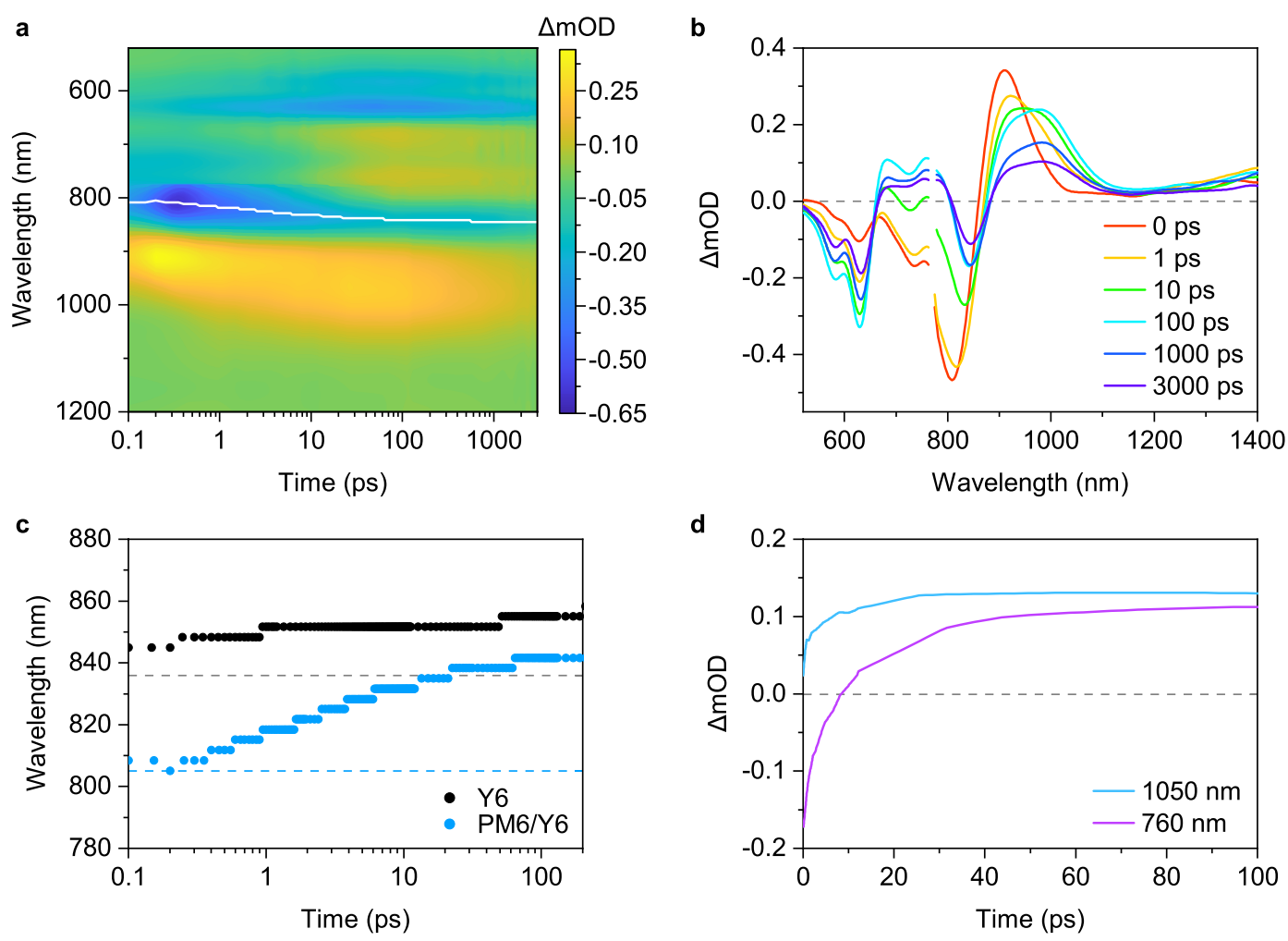

Figure 3. TA spectra of the optimized PM6/Y6 blend film. a Contour plot of the TA data and b

TA spectra of the optimized PM6/Y6 blend film. The excitation wavelength was $800 \mathrm{~nm}$ with a fluence of $1.4 \mu \mathrm{J} \mathrm{cm}^{-2}$. The white line in (a) represents the peak positions of Y6 GSB at each pump-probe delay. c Shift in the peak wavelength of Y6 GSB in the PM6/Y6 blend film (blue) as well as that in a pristine Y6 film (black) as a reference. The broken lines show the peak wavelength of respective steady-state absorption spectra. d Time evolution of TA signals monitored at 1050 $\mathrm{nm}$ (blue) and $760 \mathrm{~nm}$ (purple). 
that the rise kinetics monitored at $630 \mathrm{~nm}$ (GSB of PM6) is identical to that at $1050 \mathrm{~nm}$ (Figure S14).

Emphatically, this relatively slow exciton dissociation does not directly indicate that hole transfer is slow at the $\mathrm{D} / \mathrm{A}$ interface because this rise kinetics is a convolution of exciton diffusion to $\mathrm{D} / \mathrm{A}$ interfaces and hole transfer between Y6 and PM6 ${ }^{42,43}$. To distinguish the rate-limiting process, we focused on the TA spectra in the visible region. Importantly, the GSB signal of PM6 in the $<650 \mathrm{~nm}$ region was observed immediately after photoexcitation, indicating that Y6 excitons generated near the D/A interface undergo hole transfer within the time resolution of our TA setup. Furthermore, the rise time constant decreased with decreasing the domain size of Y6 (Figure S14). Therefore, the rise time constant of $\sim 6.0 \mathrm{ps}$ is governed by exciton diffusion to the D/A interfaces, and hole transfer between Y6 and PM6 occurs on the sub-picosecond time scale, despite the small energy offset. Hole transfer between Y6 and PM6 on the sub-picosecond time scale has also been reported previously ${ }^{18}$. This is in sharp contrast to recent observations, wherein hole transfer is considerably slow when D/A blends loose energy offset ${ }^{33,44,45}$. Fast hole transfer was also observed after photoexcitation at $900 \mathrm{~nm}$ $(1.38 \mathrm{eV})$, where excess photon energy above $E_{\mathrm{S} 1}$ is negligible (Figure $\left.\mathrm{S} 18\right)$. Therefore, the possibility of rapid hole transfer from vibronically hot Y6 excitons can be ruled out. According to Marcus theory ${ }^{46}$, fast hole transfer with a small offset is expected to require a large D-A electronic coupling and/or a small reorganization energy. Density functional theory (DFT) calculations revealed that the reorganization energy during the hole transfer is $\sim 0.27 \mathrm{eV}$ (see the Supplementary Information), 
which is considerably larger than the energy offset of this blend. Therefore, we consider that a large D-A electronic coupling is most likely a key for the fast hole transfer, which is consistent with the previous solid-state NMR measurements ${ }^{18}$, wherein the authors pointed out that the PM6 side chains and Y6 aromatic groups are in close contact in the active layer, resulting in increased D-A electronic coupling.

Interestingly, in the 700-900 $\mathrm{nm}$ region, the TA spectra changed significantly with time. Immediately after photoexcitation, we observed a negative TA signal attributable to Y6 GSB. Subsequently, this signal recovered rapidly and turned into a positive one over a $>10$ ps time scale. We identified two key features in this region. First, the rise time constant monitored at $760 \mathrm{~nm}$ was $\sim 10.7 \mathrm{ps}$, which is slightly slower than that of exciton dissociation (Figures $3 \mathrm{~d}$ and S17). In other words, a time lag exists between the exciton dissociation and the emergence of a positive PIA at $\sim 800$ $\mathrm{nm}$. Second, the peak position of Y6 GSB gradually red-shifted with time (Figures 3a and 3c). At 0 ps after photoexcitation, the peak wavelength of Y6 GSB was identical to that of the steady-state absorption $(\sim 810 \mathrm{~nm})$, whereas it finally approached $\sim 850 \mathrm{~nm}$. This final peak position is approximately equal to that of the pristine Y6 film, indicating the presence of highly ordered crystalline Y6 domains in the blend film, even though the overall crystallinity is lower than that of the pristine Y6 film, as mentioned above. This peak shift is ascribable to downhill energy relaxation of charges, which is a key driver for FC generation in this blend, as will be further discussed later. 
Impact of Y6 crystallinity on the TA spectra. In order to reveal the origin of the new PIA band in the 700-800 nm region, we also performed TA measurements on a D-rich PM6/Y6 blend film with a weight ratio of 95:5. As shown in Figure S4, the peak position of the steady-state absorption spectrum of Y6 in the D-rich blend is somewhat blue-shifted relative to that of the optimized PM6/Y6 blend film, indicating that Y6 is less aggregated in the D-rich blend film. Figure 4a shows the TA spectra of the D-rich PM6/Y6 blend film after the selective excitation of Y6 at $800 \mathrm{~nm}$. Interestingly, the Drich blend did not exhibit the positive signal around $800 \mathrm{~nm}$ and the shift in the GSB peak, which strongly suggests that both the formation of the new PIA band and the shift in the GSB peak are driven by the presence of highly ordered regions in Y6 domains.

To obtain more detailed insights, we performed TA measurements on various D/A blend films (Figures 4b-d). In the PTB7-Th/Y6 blend, dissociation of Y6 excitons occurs with a time constant of $\sim 2.0$ ps (Figures $4 \mathrm{~b}$ and $\mathrm{S} 19 \mathrm{~b}$ ), whereas the emergence of the positive PIA was as slow as $\sim 11.5 \mathrm{ps}$ (Figure S19c). These results strongly substantiate our hypothesis that the emergence of the positive PIA in the 700-800 $\mathrm{nm}$ region is not accompanied by exciton dissociation. Since the acceptor material is the same but the donor is different in the PM6/Y6 and PTB7-Th/Y6 blend films, we attribute the 
new PIA observed near $800 \mathrm{~nm}$ to the Y6 anion in crystalline domains. Interestingly, the TA spectra of the P3HT/Y6 blend film showed a clear dependence on the crystallinity of Y6, where thermal
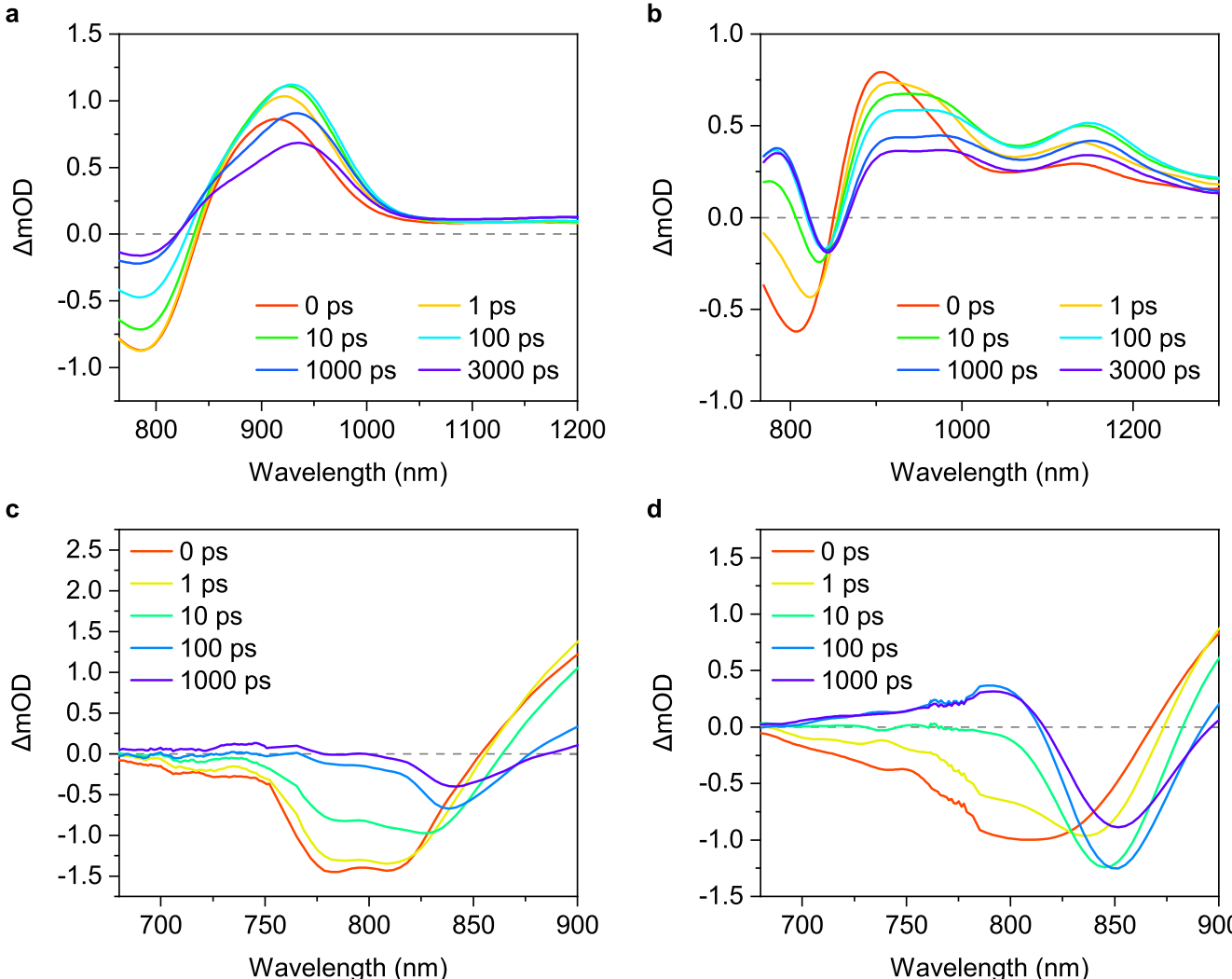

d

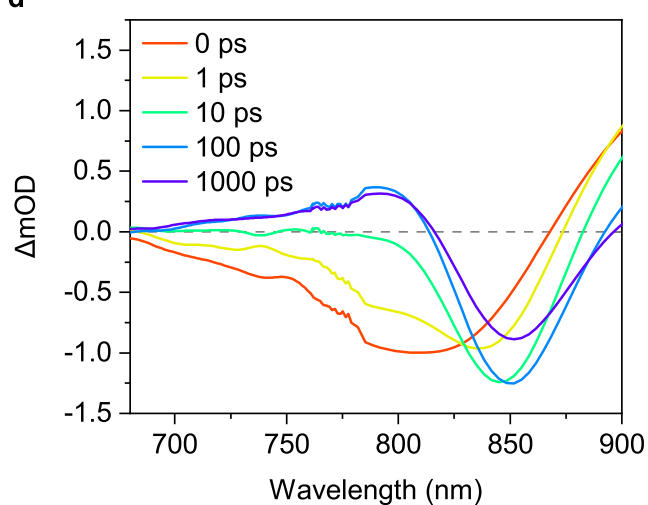

Figure 4. Impact of the crystallinity of Y6 on the TA spectra. a TA spectra of a D-rich PM6/Y6

blend film $(95: 5 \mathrm{w} / \mathrm{w})$. The excitation wavelength was $800 \mathrm{~nm}$ with a fluence of $3.2 \mu \mathrm{J} \mathrm{cm} \mathrm{cm}^{-2} . \mathbf{b}$ TA spectra of the PTB7-Th/Y6 blend film. The excitation wavelength was $800 \mathrm{~nm}$ with a fluence of $2.4 \mu \mathrm{J}$ $\mathrm{cm}^{-2}$. c,d TA spectra of a P3HT/Y6 blend film measured (c) before and (d) after thermal annealing at $140{ }^{\circ} \mathrm{C}$ for $10 \mathrm{~min}$. Full-range TA data and steady-state absorption spectra measured before and after thermal annealing can be found in the Supplementary Information. 
annealing dramatically changed the TA spectra, particularly in the 700-800 nm region (Figures 4c and 4d). The positive signal was unclear for the as-cast film, whereas it was distinct after thermal annealing. As the positive PIA is different from the hole polaron of $\mathrm{P}^{3} \mathrm{HT}^{47}$, this result reinforces our assignment of attributing the new PIA to the Y6 anion in crystalline domains. Importantly, the PIA at $\sim 800 \mathrm{~nm}$ was observed only in blend films with a relatively high EQE (Figures S9 and S10). Therefore, we conclude that the emergence of the positive PIA is a sign of efficient FC generation ${ }^{18}$.

Slow yet efficient charge dissociation. Another important finding from Figure $4 \mathrm{~d}$ is that the positive PIA observed at $\sim 700-800 \mathrm{~nm}$ is unimodal for the P3HT/Y6 blend film, in sharp contrast to the bimodal PIA for the PM6/Y6 blend film (Figure 3b). This means that the positive PIA at $\sim 680 \mathrm{~nm}$ observed in the PM6/Y6 blend film is attributable to a PM6-related signal. As shown in Figures 5a and $5 \mathrm{~b}$, the rise at $680 \mathrm{~nm}$ is slightly slower than that at $630 \mathrm{~nm}$ (GSB of PM6). The time lag between exciton dissociation and the emergence of the positive PIA at $680 \mathrm{~nm}$ is more pronounced at higher excitation fluences, at which the SSA leads to a significantly faster exciton decay (Figures S21 and $\mathrm{S} 22$ ). Therefore, the attribution of the positive PIA at $\sim 680 \mathrm{~nm}$ to the hole polaron of PM6 is inappropriate.

In line with previous studies ${ }^{18,26,28,29,37,48}$, this band is attributable to the EA of PM6. When an exciton dissociates to form an electron-hole pair at the D/A interface, the electron-hole pair generates a 
dipole-like local electric field in the surroundings. This results in a Stark shift in the absorption spectrum, resulting in the addition of a first-derivative-like transient EA to the TA spectra. As the EA amplitude depends on the strength of the local electric field, which is a function of the separation distance between the electron and hole, we can directly probe the dissociation kinetics of the electronhole pair. The fact that the EA signals reached their maximum value slightly after the occurrence of hole transfer indicates that long-range spatial dissociation of CT states takes place on a time scale of
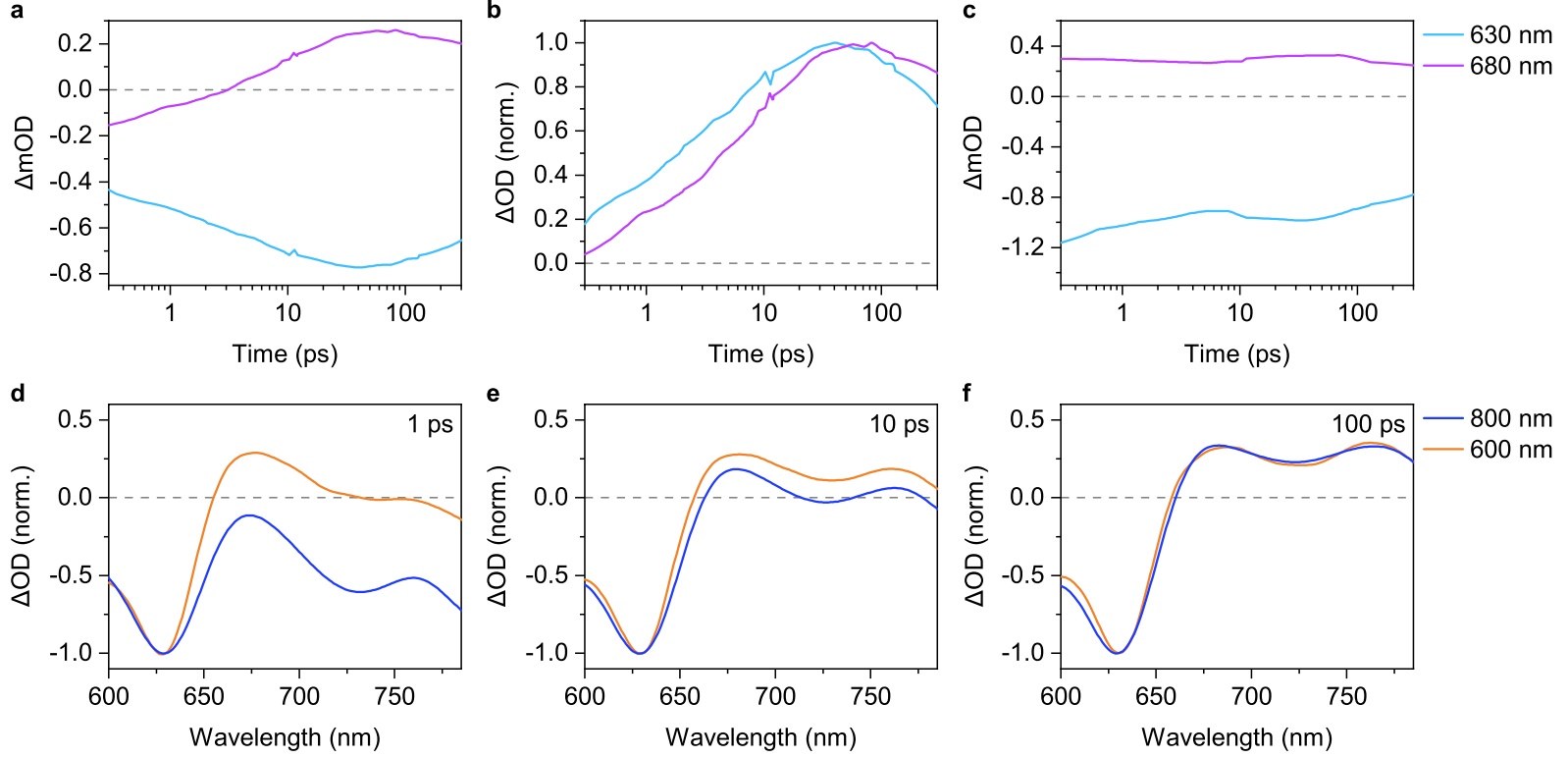

Figure 5. Time evolution of EA. a Time evolution of the TA signals monitored at $630 \mathrm{~nm}$ (PM6 GSB) and $680 \mathrm{~nm}$ (PM6 EA) excited at $800 \mathrm{~nm}$. b Normalized time evolution of panel a. c Time evolution of the TA signals monitored at $630 \mathrm{~nm}$ (PM6 GSB) and $680 \mathrm{~nm}$ (PM6 EA) excited at $600 \mathrm{~nm}$. d-f Comparison of the TA spectra after photoexcitation at $800 \mathrm{~nm}$ or $600 \mathrm{~nm}$ with a pumpprobe delay of (d) $1 \mathrm{ps,} \mathrm{(e)} 10 \mathrm{ps}$, and (f) $100 \mathrm{ps}$. TA spectra are normalized at $630 \mathrm{~nm}$ (PM6 GSB). 
picoseconds.

The situation is completely different after photoexcitation at $600 \mathrm{~nm}$, which mainly excites PM6. As shown in Figure S23, PM6 excitons dissociate into charges as rapidly as $\sim 0.5$ ps. Interestingly, the EA amplitude was already large at 1 ps after photoexcitation and almost unchanged until 100 ps (Figures 5c-f). As the offset between the excited state energy of PM6 (1.9 eV) and $E_{\mathrm{CT}}$ is sufficiently large to access the delocalized state, this fast dissociation means that electron-hole pairs undergo rapid spatial separation through the delocalized wave function before thermalization, despite the opposing Coulomb attractions, as in the case of previous studies with sufficient energy offset ${ }^{26,29}$.

These results clearly reveal that the charge dissociation mechanism after the hole transfer is different from that after the electron transfer in the PM6/Y6 blend. However, what is critically important here is that the EA amplitude after hole transfer finally reached its maximum value comparable to that after electron transfer, as shown in Figure 5f, indicating that there is no apparent difference in FC generation yield between donor and acceptor excitation. This is consistent with the flat EQE spectrum of this device (Figure S9b), indicating that FC generation yield is independent of the excitation wavelength. Efficient FC generation after Y6 selective excitation is further corroborated by the fact that charge decay dynamics is sensitively dependent on the excitation fluence (Figure S24), indicating that the bimolecular recombination is the dominant deactivation channel for charges. 
Efficient charge dissociation driven by downhill energy relaxation. The time scale of charge dissociation after the hole transfer is $\sim 10 \mathrm{ps}$, which is too slow to be rationalized by the model proposed for the large offset systems, as discussed in the previous section. On the other hand, this
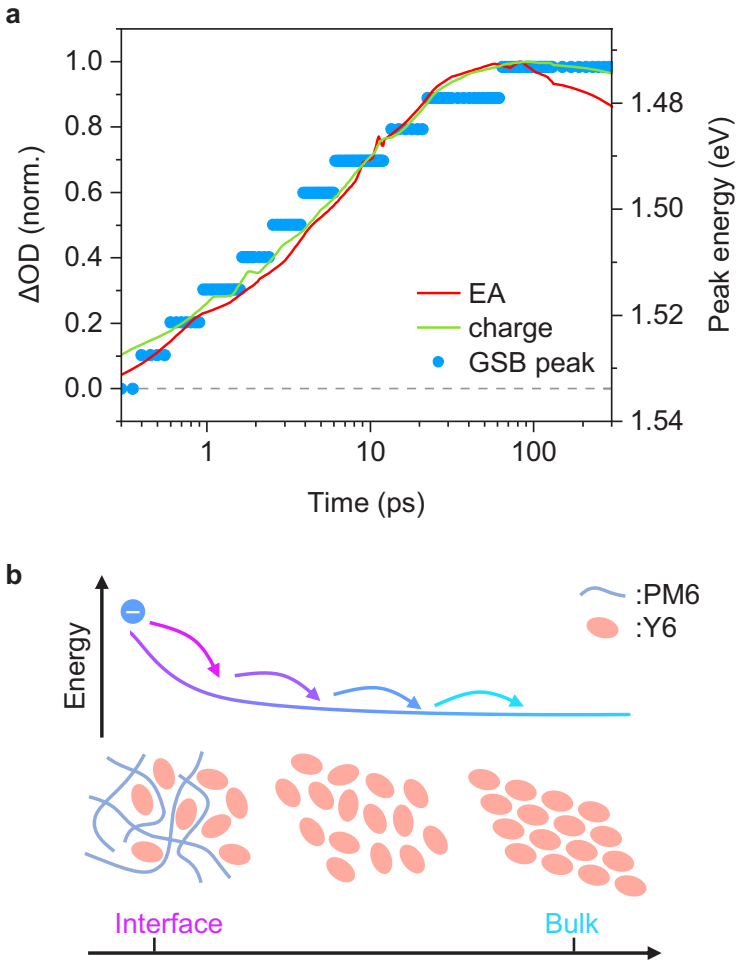

Distance from D/A interface

Figure 6. Correlation between charge dissociation and GSB peak shift. a Correlation between normalized time evolution of EA monitored at $680 \mathrm{~nm}$ and crystalline $\mathrm{Y} 6$ anion monitored at 760 $\mathrm{nm}$ (solid lines, left axis) as well as shift in the peak energy of Y6 GSB in the PM6/Y6 blend film (blue circles, right axis). b Schematic showing the relationship between the cascaded energy landscape and the crystallinity of Y6. The coexistence of less-ordered interfacial regions and highly ordered crystalline regions generates the energy cascade near the interface as a key driver for slow yet efficient FC generation. 
time scale is too fast to be rationalized within the Onsager framework ${ }^{20}$. Interestingly, as shown in Figures $6 \mathrm{a}$, the time evolution of the EA signals coincides well with that of the Y6 anion in the crystalline domain and the GSB peak shift, suggesting that the slow yet efficient charge dissociation is driven by downhill energy relaxation of charges through cascaded energy landscape near the interface. To confirm this hypothesis, we focus on the temperature dependence of the charge dissociation dynamics, as shown in Figure 7 (temperature dependence of the TA spectra is found in the Supplementary Information). Both the FC generation kinetics and the GSB peak shift were less sensitive to temperature (Figures 7a and 7b). By applying the Arrhenius fit for the inverse of the rise time constant $k_{\text {rise }}$ at $780 \mathrm{~nm}$ (Figure 7c), the activation energy for charge dissociation was determined to be as small as $\sim 9.4 \mathrm{meV}$ (this value is the upper limit, see Figure S28 for more details). As a result, the FC generation yield was clearly independent of temperature (Figure 7d). These results indicate the absence of a thermal activation barrier for charge dissociation ${ }^{17}$, which is again inconsistent with the Onsager framework but rationalized by our hypothesis that the charge dissociation is driven by downhill energy relaxation of charges through the energy cascade generated near the interfaces. Owing to the energy cascade, the Coulomb potential barrier at the interfaces is compensated, resulting in efficient charge dissociation without an activation barrier (Figures 1a and 6b). This picture also 
explains the poor FC generation in the D-rich PM6/Y6 and as-cast P3HT/Y6 blend films, wherein Y6 molecules are less aggregated; hence, the cascaded energy landscape is not formed.
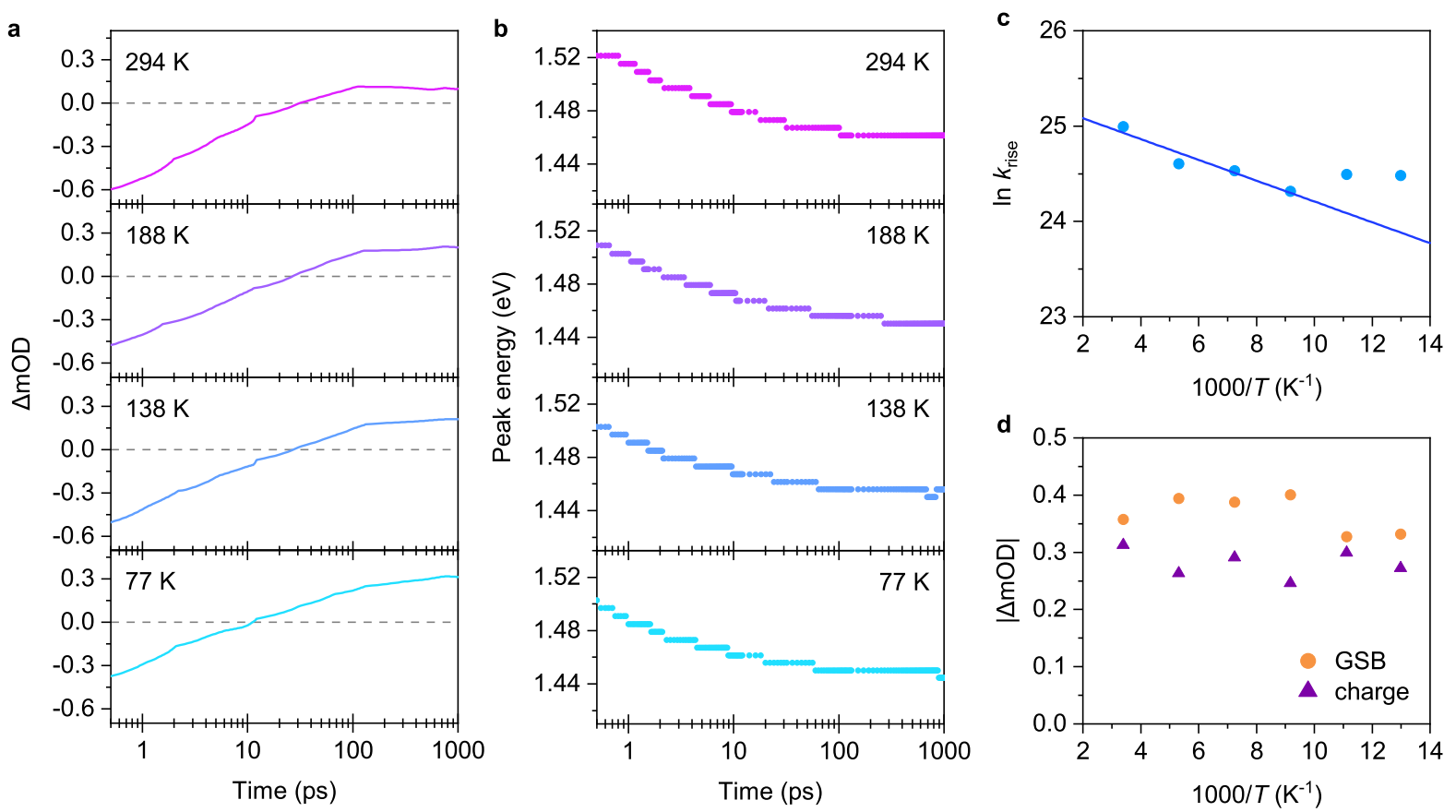

Figure 7. Temperature dependence of FC generation. a FC generation kinetics at various temperatures monitored at $780 \mathrm{~nm}$. b Shift in the peak energy of Y6 GSB at various temperatures. c Arrhenius plot for the inverse of the rise time constant $k_{\text {rise }}$ monitored at $780 \mathrm{~nm}$. The solid line represents the best fit with the Arrhenius equation using four data points on the left side. d Arrhenius plot for the TA amplitude at $850 \mathrm{~nm}$ (Y6 GSB) and $970 \mathrm{~nm}$ (charge). TA signals at 500 ps after photoexcitation are used to reduce the contribution of bimolecular recombination, which should depend on temperature. 


\section{Discussion}

The origin of this energy landscape is most likely due to the high crystallinity of Y6. Since the ordered regions are expected to be energetically more stable than at the D/A interfaces for charges, the coexistence of less-ordered interfacial regions and highly ordered crystalline regions generates the energy cascade near the interface ${ }^{49-52}$. Another possible explanation for the origin of the energy cascade is the large quadrupole moment of Y6. Recent studies have highlighted the importance of the quadrupole moment in adjusting the $E_{\mathrm{CT}}$ at the interface ${ }^{17,38,53}$, wherein the authors proposed that, since a concentration gradient of $\mathrm{Y} 6$ exists near the D/A interface, charge-quadrupole interactions increase continuously with increasing distance from the D/A interface, resulting in the cascaded energy landscape near the $\mathrm{D} / \mathrm{A}$ interface (also termed as a bias potential in their previous reports) ${ }^{17,38}$. It is clear that more effort is necessary to reveal the mechanisms underlying the formation of the energy cascade as well as understanding the desirable interfacial morphology. Nevertheless, the key for efficient FC generation without an energy offset is the presence of an energy difference between the bulk and the D/A interface. Therefore, we propose that a large discrepancy of HOMO/LUMO energy levels determined in the solution (CV) and solid (UPS or PYS) states can be used for an initial material screening for achieving efficient FC generation with a small voltage loss.

In summary, this study presents a clear, direct experimental evidence of the mechanism underlying FC generation in a state-of-the-art OSC consisting of PM6 and Y6. After the fast hole transfer from 
Y6 to PM6, despite the small energy offset of $\sim 0.1 \mathrm{eV}$, slow spatial dissociation of the CT states on a time scale of $\sim 10$ ps was observed. This is in sharp contrast to the rapid spatial separation after photoexcitation of PM6, as in the case of previous reports. Importantly, however, the slow FC generation after the hole transfer undergoes as efficient as that after the electron transfer. The activation energy for the slow charge dissociation is as small as $\sim 9.4 \mathrm{meV}$, resulting in temperature independent FC generation, even at $77 \mathrm{~K}$. We found that the cascaded energy landscape generated near the interfaces is the key driver for the slow yet efficient FC generation. Since Y6 forms an energetic cascade near the D/A interface, charges can move away from the D/A interface without experiencing the activation barrier because the attracting Coulomb barrier is compensated by the energy cascade. This study highlights the importance of the interfacial energetics for FC generation with small energy offset. Further optimization of the blend morphology will enable completely an offset-less FC generation without any geminate recombination loss.

Finally, future perspectives regarding Y6-based devices are discussed below. An unaddressed challenge regarding Y6-based devices is relatively fast bimolecular recombination loss. In the PM6/Y6 blend film, we observed the remarkable Y6 triplet formation via bimolecular recombination, as shown in Figure S25. This is probably due to the small energy difference between the lowest excited singlet and triplet states of $\mathrm{Y}^{39}$. The lowest excited triplet state energy $E_{\mathrm{T} 1}$ of $\mathrm{Y} 6$ in the solid state was estimated to be between $1.14-1.26 \mathrm{eV}$, which indicates that $E_{\mathrm{T} 1}$ is lying just below $E_{\mathrm{CT}}(1.29$ 
$\mathrm{eV})$. In the Marcus inverted regime, the CT-triplet transition rate scales exponentially with decreasing the energy difference between these states. Thus, the small CT-triplet energy difference results in a fast back charge transfer that forms Y6 triplet excitons at the D/A interfaces, which are then rapidly quenched by charges (triplet-charge annihilation) or other triplets (triplet-triplet annihilation). This means that the formation of Y6 triplet excitons is a terminal loss process. Therefore, regeneration of CT states from Y6 triplets before deactivation to the ground state will be vital for suppressing the bimolecular recombination loss.

\section{Methods}

Film preparation. Films for all spectroscopic measurements were prepared on quartz substrates, which were sequentially cleaned by sonication in toluene, acetone, and ethanol for $15 \mathrm{~min}$ each. The optimized PM6/Y6 and PTB7-Th/Y6 blends (1:1.2 w/w, donor concentration of $\left.8 \mathrm{mg} \mathrm{mL}^{-1}\right)$ were dissolved in chloroform with $0.5 \mathrm{vol} \%$ chloronaphthalene as an additive and left to stir at room temperature overnight. Thin films were spin-coated onto the cleaned substrates in a $\mathrm{N}_{2}$-filled glovebox and then thermally annealed at $110^{\circ} \mathrm{C}$ for $5 \mathrm{~min}$. Similarly, the P3HT/Y6 blend (1:1.2 w/w, donor concentration of $8 \mathrm{mg} \mathrm{mL}^{-1}$ ) was dissolved in chloroform, but without any additives. It was also stirred at room temperature overnight and spin-coated in the glovebox and then thermally annealed at $140{ }^{\circ} \mathrm{C}$ for $10 \mathrm{~min}$. The D-rich PM6/Y6 blend was prepared by the same method as the 
optimized PM6/Y6 blend, but with a D/A blend ratio of 95:5 (w/w, donor concentration of $38 \mathrm{mg} \mathrm{mL}$

${ }^{-1}$ ). For TA measurements, the samples were encapsulated in the glovebox.

Device fabrication. Photovoltaic devices were fabricated on ITO/glass substrates (Geomatec Co., $1006,10 \Omega \mathrm{sq}^{-1}$ ), which were sequentially cleaned by sonication in toluene, acetone, and ethanol for 15 min each, followed by $\mathrm{UV}-\mathrm{O}_{3}$ treatment (Nippon Laser and Electronics Lab.) for 30 min. PEDOT:PSS (Clevios, A14083) was spin-coated onto the substrates ( $\sim 30 \mathrm{~nm})$ and dried on a hot plate $\left(150^{\circ} \mathrm{C}, 20 \mathrm{~min}\right)$ under ambient conditions. Thereafter, the aforementioned active layers were spincoated in the glovebox under the same conditions. Subsequently, PDINO was spin-coated from a methanol solution $\left(2 \mathrm{mg} \mathrm{mL}^{-1}\right)$, and $\sim 80 \mathrm{~nm}$ of $\mathrm{Al}$ was thermally evaporated onto the PDINO layer. The devices were placed in a $\mathrm{N}_{2}$-filled chamber for $J-V$, EQE and EL measurements.

Steady-state absorption and emission spectra. UV-visible absorption spectra were acquired using a UV-visible spectrometer (Hitachi, U-4100). The PL spectra were measured using a fluorescence spectrophotometer (Horiba Jobin Yvon, NanoLog) equipped with a photomultiplier tube (Hamamatsu, R928P) and a liquid-N 2 -cooled InGaAs near-IR array detector (Horiba Jobin Yvon, Symphony II). The excitation wavelength was set to $790 \mathrm{~nm}$. The EL spectra were measured using the same setup with a DC voltage and current source/monitor (Advantest, R-6243). 
$\boldsymbol{J}-\boldsymbol{V}$ and EQE measurements. The $J-V$ characteristics were measured using a DC voltage and current source/monitor (Keithley, 2611B) in the dark and under AM1.5G simulated solar illumination

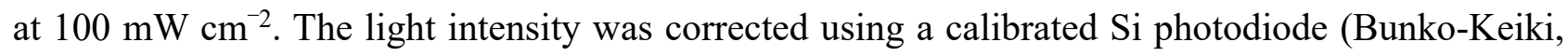
BS-520). The EQE spectra were measured using a spectral response measurement system (BunkoKeiki, ECT-25D).

CV. Materials were dissolved in acetonitrile/o-dichlorobenzene $(9: 1 \mathrm{v} / \mathrm{v})$ solutions with $0.1 \mathrm{M}$ tetrabutylammonium perchlorate as the supporting electrolyte. CV measurements were performed using a potentiostat (Princeton Applied Research, Potentiostat/Galvanostat Model 273A) at a scanning rate of $5 \mathrm{mV} \mathrm{s}^{-1}$.

PYS. The IEs were measured using a photoelectron yield spectrometer (Riken Keiki, AC-3). All films were prepared on ITO/glass substrates. The threshold energy for the photoelectron emission was evaluated on the basis of the cubic root of the photoelectron yield plotted against the incident photon energy. 
TA measurements. Femtosecond TA data were collected using a pump and probe femtosecond TA spectroscopy system, which consisted of a TA spectrometer (Ultrafast Systems, Helios) and a regenerative amplified Ti:sapphire laser (Spectra-Physics, Hurricane). A fundamental pulse with a wavelength of $800 \mathrm{~nm}$ was used as the excitation source for Y6, whereas it was converted with an ultrafast optical parametric amplifier (Spectra-Physics, TOPAS) for excitation wavelength dependence measurements. The TA data were collected over a time range from -5 ps to 3 ns. The temperature dependence was measured using a liquid-nitrogen-cooled cryostat (Oxford Instruments, Optistat DN-V).

The microsecond TA data were collected using a sensitive microsecond TA system. A Nd:YAG laser (Elforlight, SPOT-10-200-532) operating at a wavelength of $532 \mathrm{~nm}$ was used as the excitation source. White light from a tungsten lamp with a stabilized power source was used as the probe light. Two monochromators and appropriate optical cut-off filters were placed before and after the sample to increase the signal-to-noise ratio (SNR). Further details of our TA setup have been presented elsewhere ${ }^{54}$.

TA data processing. TA data were denoised by total variation (TV) regularization method to increase SNR. Details of this method are described in the Supplementary Information. 
DFT calculations. DFT calculations were performed for model compounds of isolated PM6 dimer and Y6, wherein alkyl side chains were shortened to reduce the computational effort, using the Gaussian 16 rev. A package ${ }^{55}$. In order to obtain the reorganization energy, these molecules were first optimized in the ground state using the B3LYP functional and 6-311G(d,p) basis set. Second, the positively charged PM6 dimer and negatively charged Y6 were optimized using the 6-31G(d) and 6$31+\mathrm{G}(\mathrm{d})$ basis sets, respectively. Third, TD-DFT with B3LYP/6-31+G(d) was carried out to optimize Y6 in the singlet excited state. Finally, the positively charged PM6 dimer in the ground state geometry and negatively charged Y6 in the excited state geometry were optimized using the 6-31G(d) and 6$31+\mathrm{G}(\mathrm{d})$ basis sets, respectively. The reorganization energy was then obtained from the difference of the total energy between participants.

\section{Data availability}

The data supporting the results of this work are available from the corresponding author upon reasonable request.

\section{Acknowledgements}

This study was partly supported by JST PRESTO program Grant Number JPMJPR1874, JSPS KAKENHI Grant-in-Aid for Scientific Research (B) No. 21H02012, The Murata Science Foundation, 
The Sumitomo Foundation, and Ogasawara Toshiaki Memorial Foundation.

\section{Author contributions}

Y.T. conceived and directed the project. S.N., R.S. and Y.S. performed steady-state and time-resolved optical measurements under the supervision of Y.T. T.S. and R.S. fabricated devices for all photovoltaic measurements. Y.T. developed numerical methods. S.N., T.T. and Y.T. analysed timeresolved data. S.N. and Y.T. prepared the original version of the manuscript. All authors have given approval to the final version of the manuscript.

\section{Competing interests}

The authors declare no competing interests.

\section{Additional information}

Supplementary information is available for this paper.

Correspondence and requests for materials should be addressed to Y.T. 


\section{References}

1. Hou, J., Inganäs, O., Friend, R. H., Gao, F. Organic solar cells based on non-fullerene acceptors. Nat. Mater. 2018, 17, 119-128.

2. Zhang, G. Y., Zhao, J. B., Chow, P. C. Y., Jiang, K., Zhang, J. Q., Zhu, Z. L., et al. Nonfullerene acceptor molecules for bulk heterojunction organic solar cells. Chem. Rev. 2018, 118, 3447-3507.

3. Wadsworth, A., Moser, M., Marks, A., Little, M. S., Gasparini, N., Brabec, C. J., et al. Critical review of the molecular design progress in non-fullerene electron acceptors towards commercially viable organic solar cells. Chem. Soc. Rev. 2019, 48, 1596-1625.

4. Karki, A., Gillett, A. J., Friend, R. H., Nguyen, T. Q. The path to 20\% power conversion efficiencies in nonfullerene acceptor organic solar cells. Adv. Energy Mater. 2020, 11, 2003441.

5. Armin, A., Li, W., Sandberg, O. J., Xiao, Z., Ding, L., Nelson, J., et al. A history and perspective of non-fullerene electron acceptors for organic solar cells. Adv. Energy Mater. 2021, 11, 2003570.

6. Liu, Q., Jiang, Y., Jin, K., Qin, J., Xu, J., Li, W., et al. 18\% efficiency organic solar cells. Sci. Bull. 2020, 65, 272-275.

7. Liu, F., Zhou, L., Liu, W., Zhou, Z., Yue, Q., Zheng, W., et al. Organic solar cells with 18\% efficiency enabled by an alloy acceptor: A two-in-one strategy. Adv. Mater. 2021, 33, 2100830.

8. Zhang, T., An, C., Bi, P., Lv, Q., Qin, J., Hong, L., et al. A thiadiazole-based conjugated polymer with ultradeep homo level and strong electroluminescence enables $18.6 \%$ efficiency in organic solar cell. Adv. Energy Mater., 2021, 2101705, DOI: 10.1002/aenm.202101705.

9. Zhang, M., Zhu, L., Zhou, G., Hao, T., Qiu, C., Zhao, Z., et al. Single-layered organic photovoltaics with double cascading charge transport pathways: $18 \%$ efficiencies. Nat. Commun. 2021, 12, 309.

10. Yuan, J., Zhang, Y. Q., Zhou, L. Y., Zhang, G. C., Yip, H. L., Lau, T. K., et al. Single-junction organic solar cell with over $15 \%$ efficiency using fused-ring acceptor with electron-deficient core. Joule 2019, 3, 1140-1151.

11. Karki, A., Vollbrecht, J., Dixon, A. L., Schopp, N., Schrock, M., Reddy, G. N. M., et al. Understanding the high performance of over $15 \%$ efficiency in single-junction bulk heterojunction organic solar cells. Adv. Mater. 2019, 31, 1903868.

12. Hosseini, S. M., Tokmoldin, N., Lee, Y. W., Zou, Y., Woo, H. Y., Neher, D., et al. Putting order into PM6:Y6 solar cells to reduce the langevin recombination in $400 \mathrm{~nm}$ thick junction. Sol. RRL 
2020, 4, 2000498.

13. Wu, J., Lee, J., Chin, Y.-C., Yao, H., Cha, H., Luke, J., et al. Exceptionally low charge trapping enables highly efficient organic bulk heterojunction solar cells. Energy Environ. Sci. 2020, 13, $2422-2430$.

14. Zhang, G., Chen, X.-K., Xiao, J., Chow, P. C. Y., Ren, M., Kupgan, G., et al. Delocalization of exciton and electron wavefunction in non-fullerene acceptor molecules enables efficient organic solar cells. Nat. Commun. 2020, 11, 3943.

15. Tokmoldin, N., Hosseini, S. M., Raoufi, M., Phuong, L. Q., Sandberg, O. J., Guan, H., et al. Extraordinarily long diffusion length in PM6:Y6 organic solar cells. J. Mater. Chem. A 2020, 8, 7854-7860.

16. Tokmoldin, N., Vollbrecht, J., Hosseini, S. M., Sun, B., Perdigón-Toro, L., Woo, H. Y., et al. Explaining the fill-factor and photocurrent losses of nonfullerene acceptor-based solar cells by probing the long-range charge carrier diffusion and drift lengths. Adv. Energy Mater. 2021, 11, 2100804.

17. Perdigón-Toro, L., Zhang, H., Markina, A., Yuan, J., Hosseini, S. M., Wolff, C. M., et al. Barrierless free charge generation in the high-performance PM6:Y6 bulk heterojunction nonfullerene solar cell. Adv. Mater. 2020, 32, 1906763.

18. Karki, A., Vollbrecht, J., Gillett, A. J., Xiao, S. S., Yang, Y., Peng, Z., et al. The role of bulk and interfacial morphology in charge generation, recombination, and extraction in non-fullerene acceptor organic solar cells. Energy Environ. Sci. 2020, 13, 3679-3692.

19. Phuong, L. Q., Hosseini, S. M., Sandberg, O. J., Zou, Y., Woo, H. Y., Neher, D., et al. Quantifying quasi-fermi level splitting and open-circuit voltage losses in highly efficient nonfullerene organic solar cells. Sol. RRL 2021, 5, 2000649.

20. Clarke, T. M., Durrant, J. R. Charge photogeneration in organic solar cells. Chem. Rev. 2010, 110, 6736-6767.

21. Janssen, R. A., Nelson, J. Factors limiting device efficiency in organic photovoltaics. Adv. Mater. 2013, 25, 1847-1858.

22. Gao, F., Inganäs, O. Charge generation in polymer-fullerene bulk-heterojunction solar cells. Phys. Chem. Chem. Phys. 2014, 16, 20291-20304.

23. Inganäs, O. Organic photovoltaics over three decades. Adv. Mater. 2018, 30, 1800388. 
24. Tamai, Y. Delocalization boosts charge separation in organic solar cells. Polym. J. 2020, 52, 691700 .

25. Bakulin, A. A., Rao, A., Pavelyev, V. G., Van Loosdrecht, P. H., Pshenichnikov, M. S., Niedzialek, D., et al. The role of driving energy and delocalized states for charge separation in organic semiconductors. Science 2012, 335, 1340-1344.

26. Gélinas, S., Rao, A., Kumar, A., Smith, S. L., Chin, A. W., Clark, J., et al. Ultrafast long-range charge separation in organic semiconductor photovoltaic diodes. Science 2014, 343, 512-516.

27. Jakowetz, A. C., Böhm, M. L., Zhang, J., Sadhanala, A., Huettner, S., Bakulin, A. A., et al. What controls the rate of ultrafast charge transfer and charge separation efficiency in organic photovoltaic blends. J. Am. Chem. Soc. 2016, 138, 11672-11679.

28. Jakowetz, A. C., Bohm, M. L., Sadhanala, A., Huettner, S., Rao, A., Friend, R. H. Visualizing excitations at buried heterojunctions in organic semiconductor blends. Nat. Mater. 2017, 16, 551557.

29. Tamai, Y., Fan, Y., Kim, V. O., Ziabrev, K., Rao, A., Barlow, S., et al. Ultrafast long-range charge separation in nonfullerene organic solar cells. ACS Nano 2017, 11, 12473-12481.

30. Vandewal, K. Interfacial charge transfer states in condensed phase systems. Annu. Rev. Phys. Chem. 2016, 67, 113-133.

31. Azzouzi, M., Kirchartz, T., Nelson, J. Factors controlling open-circuit voltage losses in organic solar cells. Trens Chem. 2019, 1, 49-62.

32. Vandewal, K., Mertens, S., Benduhn, J., Liu, Q. The cost of converting excitons into free charge carriers in organic solar cells. J. Phys. Chem. Lett. 2020, 11, 129-135.

33. Saito, T., Natsuda, S., Imakita, K., Tamai, Y., Ohkita, H. Role of energy offset in nonradiative voltage loss in organic solar cells. Sol. RRL 2020, 4, 2000255.

34. Wang, M., Wang, H., Yokoyama, T., Liu, X., Huang, Y., Zhang, Y., et al. High open circuit voltage in regioregular narrow band gap polymer solar cells. J. Am. Chem. Soc. 2014, 136, 12576-12579.

35. Kawashima, K., Tamai, Y., Ohkita, H., Osaka, I., Takimiya, K. High-efficiency polymer solar cells with small photon energy loss. Nat. Commun. 2015, 6, 10085.

36. Ran, N. A., Love, J. A., Takacs, C. J., Sadhanala, A., Beavers, J. K., Collins, S. D., et al. Harvesting the full potential of photons with organic solar cells. Adv. Mater. 2016, 28, 1482-1488. 
37. Menke, S. M., Cheminal, A., Conaghan, P., Ran, N. A., Greehnam, N. C., Bazan, G. C., et al. Order enables efficient electron-hole separation at an organic heterojunction with a small energy loss. Nat. Commun. 2018, 9, 277.

38. Karuthedath, S., Gorenflot, J., Firdaus, Y., Chaturvedi, N., De Castro, C. S. P., Harrison, G. T., et al. Intrinsic efficiency limits in low-bandgap non-fullerene acceptor organic solar cells. Nat. Mater. 2021, 20, 378-384.

39. Natsuda, S., Sakamoto, Y., Takeyama, T., Shirouchi, R., Saito, T., Tamai, Y., et al. Singlet and triplet excited-state dynamics of a nonfullerene electron acceptor Y6. ChemRxiv, DOI: 10.33774/chemrxiv-2021-sfwj7.

40. Vandewal, K., Tvingstedt, K., Manca, J. V., Inganäs, O. Charge-transfer states and upper limit of the open-circuit voltage in polymer:fullerene organic solar cells. IEEE J. Sel. Top. Quantum Electron. 2010, 16, 1676-1684.

41. Vandewal, K., Tvingstedt, K., Gadisa, A., Inganäs, O., Manca, J. V. Relating the open-circuit voltage to interface molecular properties of donor:acceptor bulk heterojunction solar cells. Phys. Rev. B 2010, 81, 125204.

42. Tamai, Y., Ohkita, H., Benten, H., Ito, S. Exciton diffusion in conjugated polymers: From fundamental understanding to improvement in photovoltaic conversion efficiency. J. Phys. Chem. Lett. 2015, 6, 3417-3428.

43. Umeyama, T., Igarashi, K., Sasada, D., Tamai, Y., Ishida, K., Koganezawa, T., et al. Efficient light-harvesting, energy migration, and charge transfer by nanographene-based nonfullerene small-molecule acceptors exhibiting unusually long excited-state lifetime in the film state. Chem. Sci. 2020, 11, 3250-3257.

44. Liu, Y., Zuo, L., Shi, X., Jen, A. K. Y., Ginger, D. S. Unexpectedly slow yet efficient picosecond to nanosecond photoinduced hole-transfer occurs in a polymer/nonfullerene acceptor organic photovoltaic blend. ACS Energy Lett. 2018, 3, 2396-2403.

45. Karki, A., Vollbrecht, J., Gillett, A. J., Selter, P., Lee, J., Peng, Z., et al. Unifying charge generation, recombination, and extraction in low-offset non-fullerene acceptor organic solar cells. Adv. Energy Mater. 2020, 10, 2001203.

46. Marcus, R. A. Electron transfer reactions in chemistry. Theory and experiment. Rev. Mod. Phys. 1993, 65, 599-610. 
47. Tamai, Y., Ohkita, H., Namatame, M., Marumoto, K., Shimomura, S., Yamanari, T., et al. Lightinduced degradation mechanism in poly(3-hexylthiophene)/fullerene blend solar cells. $A d v$. Energy Mater. 2016, 6, 1600171.

48. Hinrichsen, T. F., Chan, C. C. S., Ma, C., Palecek, D., Gillett, A., Chen, S., et al. Long-lived and disorder-free charge transfer states enable endothermic charge separation in efficient nonfullerene organic solar cells. Nat. Commun. 2020, 11, 5617.

49. Shoaee, S., Subramaniyan, S., Xin, H., Keiderling, C., Tuladhar, P. S., Jamieson, F., et al. Charge photogeneration for a series of thiazolo-thiazole donor polymers blended with the fullerene electron acceptors pcbm and icba. Adv. Funct. Mater. 2013, 23, 3286-3298.

50. Tamai, Y., Tsuda, K., Ohkita, H., Benten, H., Ito, S. Charge-carrier generation in organic solar cells using crystalline donor polymers. Phys. Chem. Chem. Phys. 2014, 16, 20338-20346.

51. Sweetnam, S., Graham, K. R., Ndjawa, G. O. N., Heumueller, T., Bartelt, J. A., Burke, T. M., et al. Characterization of the polymer energy landscape in polymer:fullerene bulk heterojunctions with pure and mixed phases. J. Am. Chem. Soc. 2014, 136, 14078-14088.

52. Cha, H., Fish, G., Luke, J., Alraddadi, A., Lee, H. H., Zhang, W., et al. Suppression of recombination losses in polymer:nonfullerene acceptor organic solar cells due to aggregation dependence of acceptor electron affinity. Adv. Energy Mater. 2019, 9, 1901254.

53. Schwarze, M., Schellhammer, K. S., Ortstein, K., Benduhn, J., Gaul, C., Hinderhofer, A., et al. Impact of molecular quadrupole moments on the energy levels at organic heterojunctions. Nat. Commun. 2019, 10, 2466.

54. Ohkita, H., Tamai, Y., Benten, H., Ito, S. Transient absorption spectroscopy for polymer solar cells. IEEE J. Sel. Top. Quantum Electron. 2016, 22, 100-111.

55. Frisch, M. J., Trucks, G. W., Schlegel, H. B., Scuseria, G. E., Robb, M. A., Cheeseman, J. R., et al. Gaussian 16 rev. A.01. Wallingford, CT; 2016. 